\title{
Combined FDTD/FETD Algorithm for Ground Bounce Characterization of Differential Traces through the Planes
}

\author{
Wei-Da Guo, Guang-Hwa Shiue, Chien-Min Lin , and Ruey-Beei Wu \\ Department of Electrical Engineering and Graduate Institute of Communication Engineering, \\ National Taiwan University, Taipei, Taiwan, 10617, R.O.C. \\ Tel: +886-2-23635251 x $340 \quad$ Fax: $+886-2-23638247$ \\ E-mail: rbwu@ew.ee.ntu.edu.tw \\ Advanced Assembly Division, Taiwan Semiconductor Manufacturing Co., Ltd., Taiwan, R.O.C.
}

\begin{abstract}
Based on the hybridization of finite-different and finite-element time-domain (FDTD/FETD) algorithms, a versatile local mesh refinement scheme is proposed to characterize the ground bounce of differential traces through the planes. In comparison with the conventional FDTD algorithm, the hybrid method is found to have the more computational efficiency without degrading its simulation accuracy.
\end{abstract}

\section{Introduction}

The finite-difference time-domain (FDTD) [1] algorithm has been widely used in solving for the transient responses of electromagnetic problems. While using the FDTD algorithm in its fundamental form, it is, however, difficult to model the structures that cannot fit well into Cartesian coordinates. Subsequently, a hybrid method incorporating the finite-element time-domain (FETD) algorithm into the FDTD algorithm was developed to apply for curved structures and was found to be efficient [2].

With the advent of high-speed digital era, the high-frequency noise on the DC power bus in multilayer printed circuit boards (PCBs) causes a primary concern in the signal integrity (SI) and electromagnetic interference (EMI) issues. One latent excitation mechanism of power bus noise is from the signal lines with layers changing through the via transition. In the past, the transmission-line theory and two-dimensional (2D) FDTD algorithm were linked together to simulate this problem with the single-ended via transition successfully [3]. Due to the higher noise immunity and EMI reduction, differential signaling has become a popular wiring approach for high-speed data rate interconnects. Nevertheless, for the actual layout constraints, the common-mode current may be generated from various imbalances in the circuit, such as the driver-phase skew, termination varieties, signal-path asymmetries, etc. Both differential-mode and common-mode currents can also excite the DC power bus, resulting in the ground bounce propagating within the planes seriously.

In use of the conventional method to deal with this case, it will need the finer mesh to accurately model the close signals transitioning through the parallel plates and take the more simulation time. To increase the computational efficiency, this paper expands the application of FETD algorithm for the region with close signal transitions, while the other regions are modeled by FDTD algorithm as well. Finally, the simulation results are compared with those simulated by CST Microwave Studio [4] to verify its accuracy.

\section{Simulation Methodology}

A typical differential via transition in a four-layer board is shown in Fig. 1. Along the signal flow path, the whole structure can be divided into three parts: (1) the coupled transmission line, (2) the via discontinuities, and (3) the parallel plates. Given the even and odd mode propagation constants and characteristic impedances, the coupled transmission lines are modeled by the equivalent $L C$ ladder circuit and the signal propagation can be simulated by the telegrapher's equations using the

The work was supported in part by the National Science Council, Republic of China, under Grant NSC 91-2213-E-002-109, Ministry of Education under Grant 93B-40053, and TSMC under Grant 93-FS-B072. 
central difference discretization. By introducing the layer peeling process, via discontinuities are separated into three segments which take two solid planes as the natural boundaries and each segment can be modeled as a SPICE passive network with reference to the full-wave simulation in [5]. Since the separation between the two solid planes is much less than the equivalent wavelength of signal, the electromagnetic fields inside can be assumed to be uniform along the vertical direction and solved by the 2D FETD/FDTD algorithm.

The FETD algorithm starts from the Maxwell's two-curl equations and the vector equation can be obtained by

$$
\nabla \times \nabla \times \vec{E}+\mu \varepsilon \frac{\partial^{2} \vec{E}}{\partial t^{2}}+\mu \frac{\partial \vec{J}}{\partial t}=0 \text { in } \Omega,
$$

where $\vec{E}$ and $\vec{J}$ denotes the electric field and current density, respectively, in the volume $\Omega$. Applying the weak formulation or the Galerkin's procedure to Eq. (1) gives

$$
\int_{2}(\nabla \times \vec{W} \bullet \nabla \times \vec{E}) d \Omega+\mu \varepsilon \int_{2} \vec{W} \bullet \frac{\partial^{2} \vec{E}}{\partial t^{2}} d \Omega+\mu \int_{2} \vec{W} \bullet \frac{\partial \vec{J}}{\partial t} d \Omega+\frac{\mu_{r}}{c} \oint_{\infty} \vec{W} \bullet\left(\frac{\partial \vec{E}}{\partial t} \times \hat{n}\right) d \Gamma=0,
$$

where $\vec{W}$ is the test function that can be arbitrarily defined. The variational formula should be discretized to proceed with the numerical computation. By the finite-element method, the suitable basis functions are chosen to express the field inside each triangular element. After taking volume integration inside each element and assembling the integrals from all the elements, Eq. (2) can be simplified into a matrix form as read by

$$
[C] \frac{\partial^{2} E}{\partial t^{2}}+[B] \frac{\partial E}{\partial t}+[D] E+[F] \frac{\partial J}{\partial t}=0,
$$

where $E$ and $J$ are the coefficient vectors of electric field and current density, respectively, Besides, the values of all matrix elements are formulated as the follows:

$$
\begin{aligned}
& {[C]_{i j}=\mu \varepsilon \int_{2} \vec{W}_{i} \bullet \vec{W}_{j} d \Omega,[B]_{i j}=\frac{\mu_{r}}{c} \oint_{x} \vec{W}_{i} \bullet\left(\vec{W}_{j} \times \hat{n}\right) d \Omega,} \\
& {[D]_{i j}=\int_{2} \nabla \times \vec{W}_{i} \bullet \nabla \times \vec{W}_{j} d \Omega, \text { and }[F]_{i}=\mu \int_{2} \vec{W}_{i} d \Omega .}
\end{aligned}
$$

For the meshing profile as demonstrated in Fig. 2, the FETD region is chosen to be a block replacing the original FDTD region into which the via transition penetrates. Consequently, the electric-field values of interface calculated from the FDTD algorithm become the Dirichlet boundary conditions of the FETD region. The flow chart as described in Fig. 3 by simulating the telegrapher's equations of coupled transmission lines simultaneously is proposed to import the updated parameters into the FETD region and thereof solve for the unknown electric fields at the next time step to characterize the ground bounce.

\section{Numerical Results}

Consider the original structure in Fig. 1 as an example. The size of the PCB in the $x-y$ plane is $10 \mathrm{~cm} \times 10 \mathrm{~cm}$ and the separation between the two solid planes is $0.5 \mathrm{~mm}(20$ mil $)$ thick. The centric locations of the via transitions of differential signals are at $(5 \mathrm{~cm}, 4.93 \mathrm{~cm})$ and $(5 \mathrm{~cm}, 5.07 \mathrm{~cm})$, while a probe is located at $(5 \mathrm{~cm}, 6 \mathrm{~cm})$ to measure the ground bounce voltage induced on the planes. Fig. 4 depicts the physical dimensions of the coupled transmission lines and via discontinuities. The voltage sources are Gaussian pulse generators driving the differential signals with the rise time of $100 \mathrm{ps}$ and amplitude of $\pm 2 \mathrm{~V}$. On the ends of signal lines are all connected with the matched loads.

When the perfect magnetic conductor (PMC) is used for the boundary condition of parallel-plate structure, Fig. 5 and Fig. 6 present the simulated waveforms with and without the signal skew at the pre-allocated probe, respectively. The voltage fluctuation before $700 \mathrm{ps}$ is induced by the incident signal passing through the via discontinuities, while the late-time response is due to the generation of 
plane resonance. It is shown that there is a good agreement between the results by the proposed FDTD/FETD algorithm, conventional FDTD algorithm, and CST Microwave Studio. Moreover, the voltage level of late-time response in Fig. 5 is greater than that in Fig. 6 because of the existence of common-mode current produced by the time skew of differential signal.

If only the FDTD algorithm is chosen to identify the region with close signal transitions, the smaller grids are required in order to obtain the accurate resolution. By locally applying the FETD grids to the specified region, the necessary resolution is also given without the need to refine the whole space with the smaller cell size. From Table I, it is found that the run time of FDTD/FETD algorithm is much less than that of the conventional FDTD algorithm [6]. As a result, the injection of the FETD grids into the FDTD region can reduce required memory and run time while also providing the accuracy comparable to a single FDTD mesh with the smaller cell size.

\section{Conclusion}

A hybrid 2D time-domain method is introduced in this paper and applied to characterize the ground bounce due to the differential via transition in the multilayer PCBs. This method hybridizes the efficient FDTD analysis for most of the regular region with a flexible FETD analysis for the region having differential via transition inside. It is demonstrated that the computational time and memory requirements are far less than those of an FDTD space with the smaller mesh resolution throughout. Furthermore, while the signal rise-time becomes much smaller nowadays, a little time skew of differential signals may lead to a great level of common-mode current. The appearance of common-mode current will deteriorate the system reliability and should be carefully considered in the PCB layout design.

\section{References}

[1] K. S. Yee, "Numerical solution of initial boundary value problems in isotropic media," IEEE Trans. Antennas Propagat., vol. AP-14, pp. 302-307, May 1966.

[2] R. B. Wu and T. Itoh, "Hybrid finite-difference time-domain modeling of curved surfaces using tetrahedral edge elements," IEEE Trans. Antennas Propagat., vol. 45, pp. 1302-1309, Aug. 1997.

[3] S. M. Lin and R. B. Wu, "Composite effects of reflections and ground bounce for signal vias in multi-layer environment," Proc. IEEE Microwave Conf. APMC, vol.3, pp. 1127-1130, Dec. 2001.

[4] CST Microwave Studio Manual, Computer Simulation Technology, Germany, 2003 (www.cst.com).

[5] J. Fan, J. L. Knighten, N. W. Smith, and R. Alexander, "Extraction of a SPICE via model from full-wave modeling for differential signaling," Proc. IEEE Int.Symp. Electromagn. Compat., vol.2, pp. 577-582, Aug. 2004.

[6] Simulation Package for Electrical Evaluation and Design (SpeedXP), Sigrity Incorporated, Santa Clara, CA (www.sigrity.com).

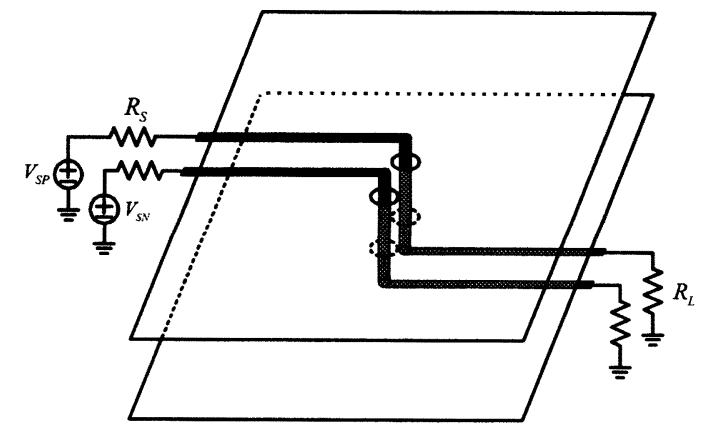

Fig. 1. A typical differential via transition in a four-layer board.

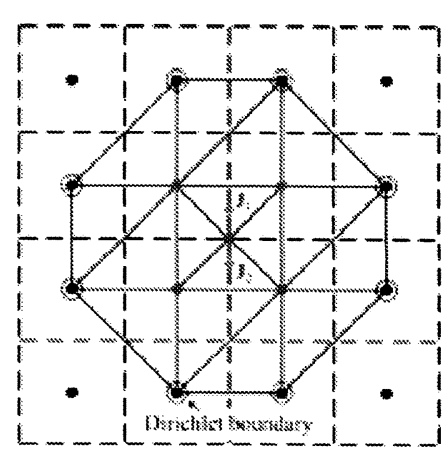

Fig. 2. FEM mesh in source region and its interface with FDTD grid. 


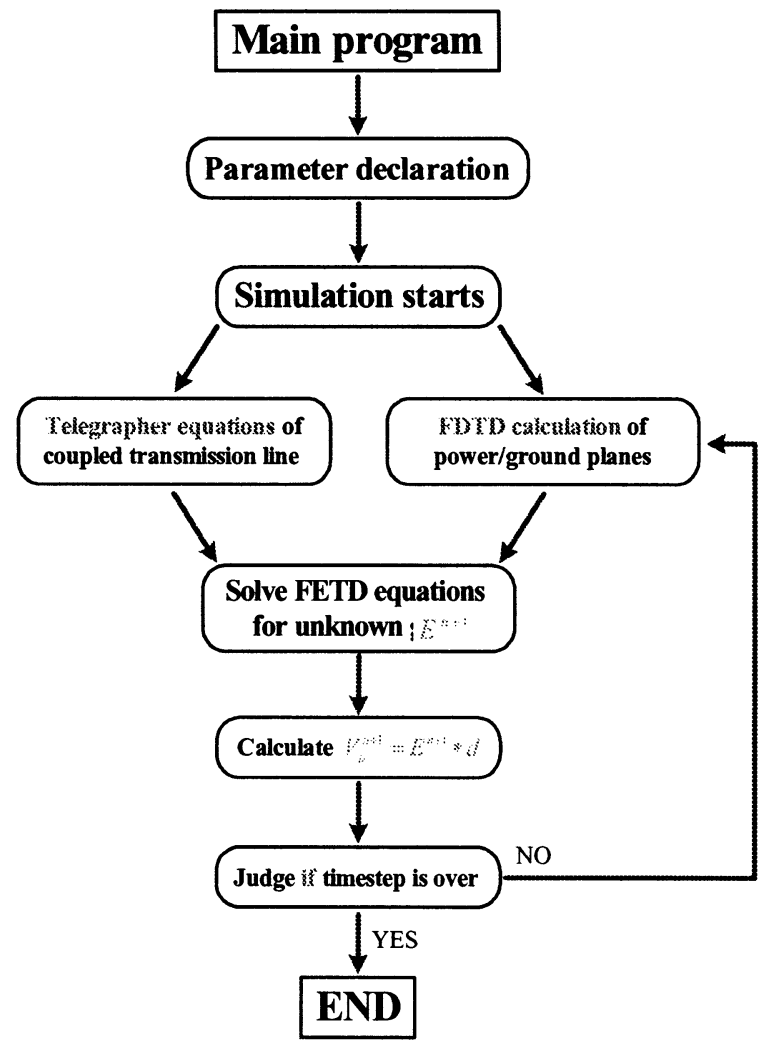

Fig. 3. A flow chart of FDTD/FETD algorithm for the ground bounce characterization.

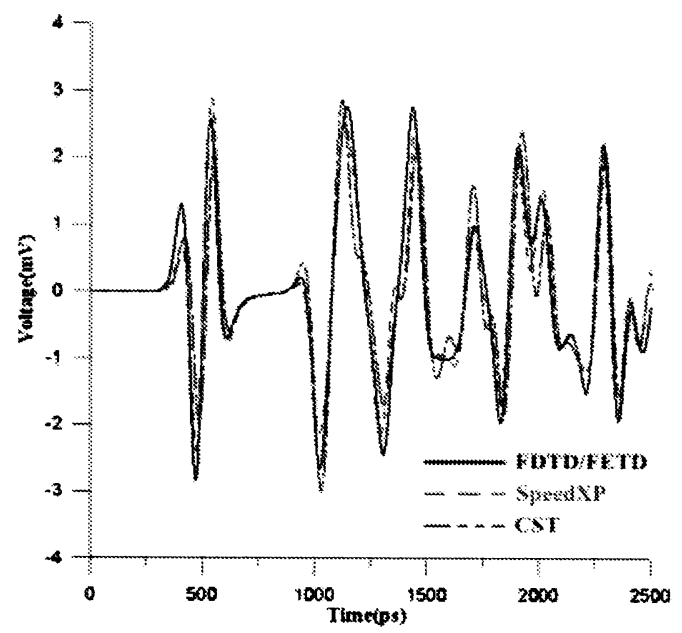

Fig. 5. Simulated waveforms of the ground bounce with the signal skew of 10 ps.

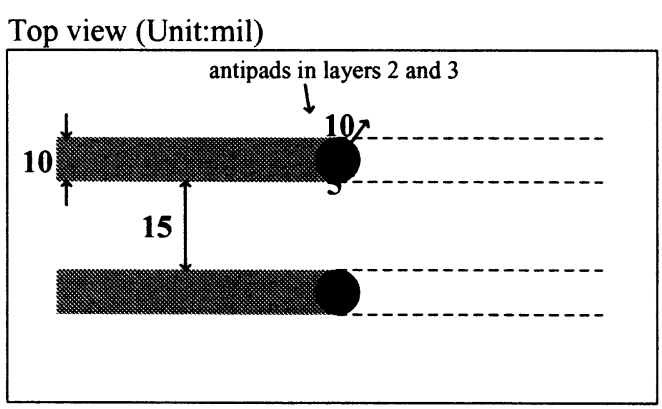

(a) Top view.

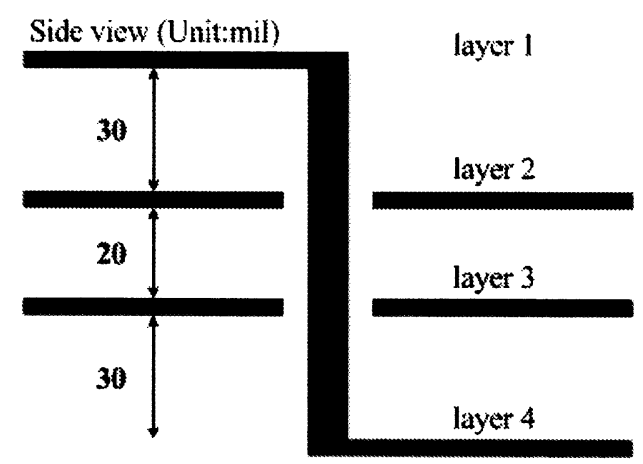

(b) Side View.

Fig. 4. Physical dimensions of Fig. 1.

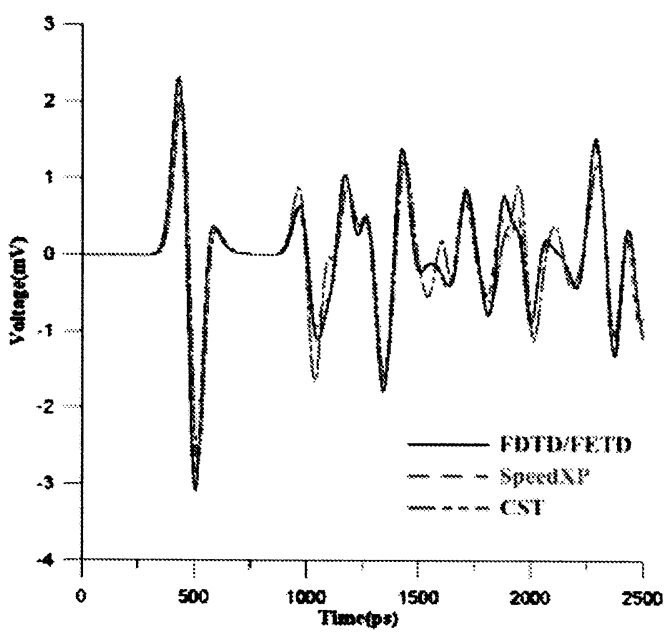

Fig. 6. Simulated waveforms of the ground bounce without the signal skew.

Table I. Run time of the ground bounce simulation by using three methods.

\begin{tabular}{|c|c|c|c|}
\hline Scheme / Tool & $\begin{array}{c}\text { Proposed FDTD/FETD } \\
(\mathrm{dx}=\mathrm{dy}=1.25 \mathrm{~mm}, \mathrm{dt}=5 \mathrm{ps})\end{array}$ & $\begin{array}{c}\text { SpeedXP } \\
(\mathrm{dx}=\mathrm{dy}=0.62 \mathrm{~mm}, \mathrm{dt}=2.5 \mathrm{ps})\end{array}$ & $\begin{array}{c}\mathrm{CST} \\
(\text { full-wave analysis })\end{array}$ \\
\hline Run Time & $0.9 \mathrm{sec}$ & $16.2 \mathrm{sec}$ & more than 1 hour \\
\hline
\end{tabular}

